\title{
marges Marges
}

revue d'art contemporain Revue d'art contemporain

\section{2 | 2016}

\section{L'Artiste-théoricien}

\section{Didier Faustino, Des Corps \& Des Astres}

Le Magasin, Grenoble, 12 septembre 2015 - 3 janvier 2016

\section{Umut Ungan}

\section{CpenEdition}

\section{Journals}

Édition électronique

URL : http://journals.openedition.org/marges/1154

DOI : $10.4000 /$ marges. 1154

ISSN : 2416-8742

Éditeur

Presses universitaires de Vincennes

\section{Édition imprimée}

Date de publication : 22 avril 2016

Pagination : 182-183

ISBN : 978-2-84292-529-1

ISSN : 1767-7114

Référence électronique

Umut Ungan, «Didier Faustino, Des Corps \& Des Astres », Marges [En ligne], 22 | 2016, mis en ligne le 22 avril 2016, consulté le 25 septembre 2020. URL : http://journals.openedition.org/marges/1154 ; DOI : https://doi.org/10.4000/marges.1154 


\section{«Des Corps \& Des Astres. Didier Faustino »}

Le Magasin, Grenoble

12 septembre 2015 - 3 janvier 2016
S'il est toujours possible d'appréhender une exposition uniquement pour ce qu'elle donne à voir au visiteur, une connaissance plus élargie des conditions de fabrication qui mène au produit final n'en affecte pas moins, positivement ou négativement, son évaluation globale. L'exposition monographique de Didier Faustino au centre d'art contemporain le Magasin semble illustrer ce type de cas. Le mouvement de grève des employés en septembre dernier - qui a mis à la lumière du jour les interactions difficiles et les rapports de force au sein de l'institution grenobloise entre son personnel, son directeur et sa présidente du conseil d'administration - bien qu'il reste un élément externe à l'appréciation proprement esthétique de l'exposition de Faustino, influe néanmoins sur la forme que prend cette dernière, aussi bien sur sa visibilité que sur sa lisibilité. Hormis les conséquences techniques de la "perturbation» liée au mouvement (fermeture du centre, retard/annulation des projets en cours et à venir), il s'agit avant tout d'une situation particulière, puisque «Des Corps \& Des Astres » relève d'une programmation de «secours » (comme celle de l'exposition précédente, «Pression Liberté Expression » de Dan Perjovschi), l'architecte/ artiste étant choisi directement par le CA en l'absence du directeur, en arrêt maladie suite aux évènements. Un tel « dépassement » de la fonction administrative, s'il peut être motivé, entre autres, par une volonté de poursuivre le bon déroulement de l'offre artistique de l'institution, modifie inévitablement cette dernière sur le plan qualitatif. À la relative homogénéisation des propositions (Faustino et Perjovschi étant tous les deux représentés par la galerie Michel Rein) s'ajoutent des effets sur le contenu même de l'exposition. Bien que la présentation insiste à la fois sur des «œuvres créées après 2007 ainsi que des installations monumentales spécifiquement pensées pour le Magasin », « Des Corps 
\& Des Astres » propose un parcours relativement réduit du travail de Faustino, avec seulement neuf œuvres qui vont de 2007 à 2015 . Malgré le fait que l'on puisse interpréter ce choix comme une certaine lecture "indéterminée » de son travail (comme semble indiquer Nowhere Somewhere [2013], l'œuvre au plafond de la salle introductive de l'exposition, avec ses flèches en néon qui indiquent des directions entremêlées) et non comme une rétrospective historique, cette réduction est d'autant plus ressentie qu'elle est mise en scène dans l'étendue considérable que constitue l'espace d'exposition du Magasin. Du côté des installations in situ, mises en avant sur le plan textuel probablement pour appuyer l'idée d'un investissement véritable des lieux (et non d'une organisation hâtive et arbitraire) par l'artiste, elles ne sont qu'au nombre de deux: Vortex Populi (2015), un tourbillon spectaculaire de barrières Vauban placée dans la Rue, la partie immédiatement visible par le visiteur qui entre dans le centre d'art avant même d'accéder aux salles et à Ashes to Ashes (2015), ensemble d'étoiles en béton disposées au sol, renvoyant au Walk of Fame hollywoodien, augmentées de l'inscription habeas corpus (" sois maître de ton corps »). II s'agit en réalité d'une œuvre originairement destinée à la Biennale de La Havane en 2015 (avec le titre The Dark Side of the Moon et l'inscription Soy Unico, "je suis unique » sur les mêmes étoiles en question) et qui s'est vue refusée par les autorités cubaines, comme en informe le texte de la commissaire "invitée » Reiko Setsuda, chargée de la programmation du Forum de la Maison Hermès à Tokyo (où Faustino a également exposé en 2010) et ancienne élève de l'École du Magasin. Il est étonnant de voir que ce « remaniement » formel du projet initial, s'il relève d'une pratique habituelle mais faisant partie de « l'arrière-plan » de l'activité artistique, est explicité et assumé dans le texte de la commissaire, affaiblissant par là la force de l'argument de l'investissement que représente le travail spécifique in situ, avec donc un projet qui n'était pas pensé au départ pour le Magasin. Dans l'ensemble, le dialogue entre les œuvres est suggéré par des liens que tisse un thème/concept assez abstrait qu'est le « corps »: celui de l'artiste avec (G)host in the (S)hell (2008); celui cultivé mais absent dans la pierre tombale augmentée d'une haltère de Lost Last Lust (2007); celui évoqué par les articulations des sculptures gonflables de Décors \& Désastres (2013) réalisées pour le chorégraphe Richard Siegal, pour n'en citer que quelques-uns. Si ce thème traverse effectivement la pratique artistique/architecturale de Faustino, il devient ici un outil conceptuel bien trop accommodant pour relier les différents supports (photo, sculpture, installation) mais aussi pour la glose, comme en témoigne le texte de Reiko Setsuda. Titré «fragments pour un point intermédiaire stable », ce dernier est composé de cinq "séquences» qui forment des "notes» avec des descriptions d'œuvres et interprétations «qui ne doivent pas être retenues comme une critique définitive ». À la fois dans la position du visiteur «naîf », du poète inspiré et du critique avisé, son discours vacille entre les plans phénoménal, interprétatif et historicisant des œuvres, en une écriture qui reste assez représentative de la structure de l'exposition «d'urgence». Au détriment d'une certaine profondeur, elle fait de la suggestion et de l'abstraction ses point focaux, seules conditions pour une cohérence de l'ensemble, mais dépouillant par là l'efficacité expérientielle des œuvres et de la démarche artistique de Faustino, qui ne manque pas pourtant d'ancrer sa pratique dans la société actuelle.

\section{Umut Ungan}

